\title{
SQUID Developments for the Gravitational Wave Antenna MiniGRAIL
}

\author{
J. Pleikies, O. Usenko, K. H. Kuit, J. Flokstra, A. de Waard, and G. Frossati
}

\begin{abstract}
We designed two different sensor SQUIDs for the readout of the resonant mass gravitational wave detector MiniGRAIL. Both designs have integrated input inductors in the order of $1.5 \mu \mathrm{H}$ and are planned for operation in the $\mathrm{mK}$ temperature range. Cooling fins were added to the shunt resistors. The fabricated SQUIDs show a behavior that differs from standard dc-SQUIDs. We were able to operate a design with a parallel configuration of washers at reasonable sensitivities. The flux noise saturated to a value of $0.84 \mu \Phi_{0} / \sqrt{ } \mathrm{Hz}$ below a temperature of $200 \mathrm{mK}$. The equivalent noise referred to the current through the input coil is $155 \mathrm{fA} / \sqrt{ } \mathrm{Hz}$ and the energy resolution yields $62 \hbar$.
\end{abstract}

Index Terms-Gravitational wave antenna, SQUIDs, superconducting devices.

\section{INTRODUCTION}

$\mathbf{T}$ HE gravitational wave detector MiniGRAIL is the first spherical resonant mass detector. When excited by a gravitational wave, the $\mathrm{CuAl} 6 \%$ sphere with a diameter of $68 \mathrm{~cm}$ is expected to show variations in diameter of the order of $10^{-20} \mathrm{~m}$ at its resonance modes around $3 \mathrm{kHz}$ [1]. To reach the highest sensitivity, the aimed operation temperature is $20 \mathrm{mK}$.

For the readout of the MiniGRAIL, a capacitive transducer was developed which converts the displacement into a current that has to be measured by a highly sensitive readout system. Because of its low-noise properties, direct current superconducting quantum interference devices (dc-SQUIDs) were chosen to measure this displacement current.

Compared to standard SQUIDs, there are a few special requirements that have to be watched carefully. First of all, a tightly coupled integrated coil is needed. Because of the capacitive load and signal frequencies in the $\mathrm{kHz}$ range, the SQUID needs a high input inductance, here about $1.5 \mu \mathrm{H}$, that still has to be matched to the capacitive transducer, using an inductive transformer.

To reach the best sensitivity, the hot-electron effect has to be considered. Due to a weakened electron-phonon coupling at temperatures below typically $1 \mathrm{~K}$, the heat dissipated in the shunt resistors leads to a limitation of the minimum operation temperature of the SQUID and connected to it to a minimum reachable noise level. Cooling fins can be attached to reach lower minimum operation temperatures [2].

Manuscript received August 28, 2006. This work was supported in part by Stichting Technische Wetenschappen (STW).

J. Pleikies, K. H. Kuit and J. Flokstra are with the Low Temperature Division, Faculty of Science and Technology, University of Twente, P.O. Box 217, 7500 AE Enschede, the Netherlands (e-mail: j.pleikies@tnw.utwente.nl).

O. Usenko, A. de Waard and G. Frossati are with the Kamerlingh Onnes Laboratory of the Leiden University, P.O. Box 9504, 2300 RA Leiden, the Netherlands (e-mail: usenko@physics.leidenuniv.nl).

Digital Object Identifier 10.1109/TASC.2007.898067

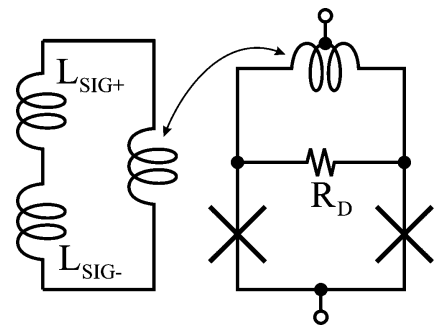

Fig. 1. Schematic of the dc-SQUID with integrated flux transformer. For simplicity, the shunt resistors and the coils coupling to the gradiometric signal washers $L_{S I G}$ are not shown. The integrated coils are on-chip connected in series. The feedback flux can be applied via a single wire around the SQUID.

Furthermore, resonant mass antennas have to be treated as a resonant load with a high quality factor, requiring that the noise power reflected from the readout system is as small as possible. Besides a standard dc-SQUID with an integrated flux transformer, a design with a minimum direct mutual inductance between the feedback and the signal coil systems was made. This is believed to reduce back-action effects in flux-locked loop (FLL) operation [3].

In this paper we focus on the design steps taken to develop new sensor dc-SQUIDs for the readout of MiniGRAIL and on the first measurement results.

\section{DESCRIPTION OF THE DESIGN PROCESS}

We made designs for a process with $110 \mathrm{~A} / \mathrm{cm}^{2}$ critical current density at the JeSEF foundry [4] with the possibility to add cooling fins to the shunt resistors. The layer of the shunt resistors is Palladium-Gold, whereas the cooling fins are realized in a layer of Gold with a thickness of $100 \mathrm{~nm}$. There are two Niobium layers available. The two designed sensor SQUIDs are dc-SQUIDs with a multiple washer configuration and an integrated flux transformer.

According to our earlier designs [5] and their measured behavior, we focused during this design step on the weakening of negative effects due to resonances in the coil- and washerstructures.

\section{A. dc-SQUID With Integrated Flux Transformer}

The dc-SQUID with cooling fins is coupled to a gradiometric coil system, as shown in Fig. 1.

The critical current of the SQUID $2 I_{0}$ is $22.5 \mu \mathrm{A}$. The hysteresis of the junctions is removed by resistive shunts of $5.7 \Omega$ for each junction. The calculated McCumber parameter $\beta_{C}$ is 0.71 .

The characteristic inductances were estimated taking into account the screening of the transformer loop. The SQUID inductance $L_{S Q}$ is $128 \mathrm{pH}$, yielding to a screening parameter $\beta_{L}$ 


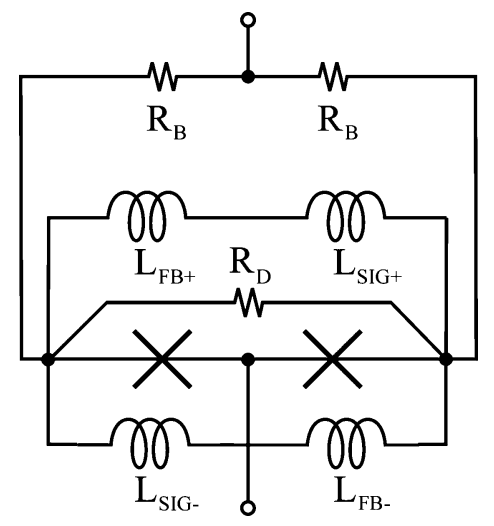

Fig. 2. Schematic of the dc-SQUID with a parallel washer configuration. For simplicity, the shunt resistors in parallel to the Josephson junctions and the coils coupling to the signal- and feedback-washers $\left(L_{S I G}\right.$ and $\left.L_{F B}\right)$ are not shown. The integrated coils are on-chip connected in series. The washers are in a gradiometric configuration.

of 1.39. The effective inductance of the signal coil system is $1.58 \mu \mathrm{H}$ with a mutual inductance $M$ of $10.4 \mathrm{nH}$ to the SQUID inductance.

A guess for the best reachable sensitivity can be done with the theory for non hysteretic, uncoupled dc-SQUIDs [6], [7]. The directly measurable value is the flux noise $S_{\Phi}$, the noise power spectrum density (PSD) of the SQUID and the readout system referred to the SQUID inductance. In case of SQUIDs with integrated input coils, it is useful to refer the noise level to the input current $S_{I}=S_{\Phi} / M^{2}$. The common figure of merit for comparing SQUIDs with differing SQUID inductance is the energy resolution $\varepsilon=S_{\Phi} /\left(2 \cdot L_{S Q}\right)$, which represents the white noise energy per bandwidth referred to the SQUID inductance.

For this design we calculated for a temperature of $4.2 \mathrm{~K}$ an energy resolution of $73 \hbar$ or a flux noise amplitude spectrum density (ASD) $\sqrt{ } S_{\Phi}$ of $0.68 \mu \Phi_{0} / \sqrt{ } \mathrm{Hz}$, respectively. $\Phi_{0}$ is the magnetic flux quantum of $2.07 \cdot 10^{-15} \mathrm{~Wb}$.

To overcome resonance effects, we used a resistor shunting the washer (washer resistance, $R_{D}$ in Fig. 1) with the same value as the shunt resistors of the Josephson junctions $(5.7 \Omega)$.

\section{B. dc-SQUID With a Parallel Washer Configuration}

The design is a modified version of a commercial available Quantum Design chip [8]. Because of its highly symmetric layout, a direct mutual inductance between the feedback coil system and the signal inductances are reduced to a minimum. The main differences are added cooling fins and the splitting of the bias current is done on-chip via the two parallel resistors $R_{B}$ as indicated in Fig. 2 .

The critical current of the SQUID $2 I_{0}$ is $22.5 \mu \mathrm{A}$. With the $5.6 \Omega$ resistance connected in parallel to the junctions, the McCumber parameter $\beta_{C}$ was estimated to be 0.67 .

The calculated SQUID inductance $L_{S Q}$ of $230 \mathrm{pH}$ yields a screening parameter $\beta_{L}$ of 2.5 . The effective inductance of the signal coil system is $1.45 \mu \mathrm{H}$ with a mutual inductance $M$ of $12.2 \mathrm{nH}$ to the SQUID inductance.

At $4.2 \mathrm{~K}$ we estimated the optimum sensitivity [6], [7] of the design in terms of energy resolution $\varepsilon$ to $89 \hbar$ or a flux noise ASD $\sqrt{ } S_{\Phi}$ of $1.0 \mu \Phi_{0} / \sqrt{ } \mathrm{Hz}$, respectively.
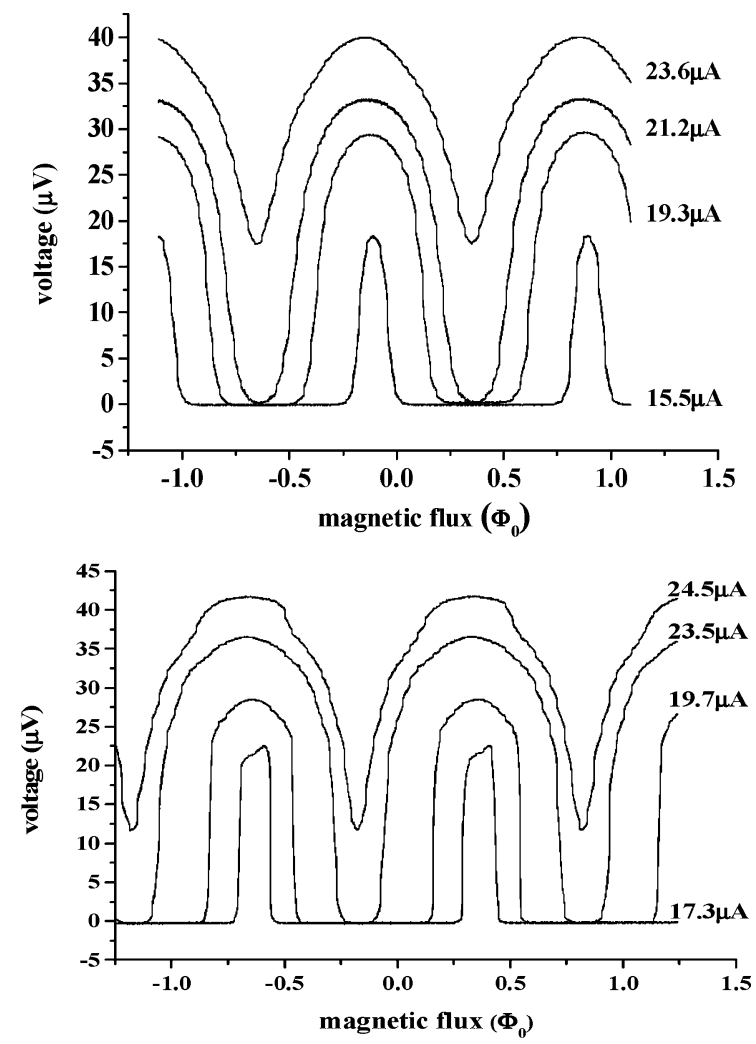

Fig. 3. Characteristics measured with sweeping magnetic flux in single stage with current bias at $4.2 \mathrm{~K}$ (upper) and at $0.3 \mathrm{~K}$ (lower). The applied bias currents are indicated on the right side of the graphs. A constant flux bias was applied. The signal coil was shunted with a resistor of $30 \Omega$ and a capacitor of $1 \mathrm{nF}$ in series. The maximum flux-to-voltage transfer was estimated to be $270 \mu \mathrm{V} / \Phi_{0}$ at $4.2 \mathrm{~K}$ and $820 \mu \mathrm{V} / \Phi_{0}$ at $0.3 \mathrm{~K}$.

We used a resistor in parallel to the washer with the same value as the shunt resistors $(5.6 \Omega)$ to damp resonances.

\section{RESUlts OF THE CHARACTERIZATION}

The fabricated samples show in both the critical current density and the sheet resistance good agreement with the values used for the design.

\section{A. Description of the Characterization Process}

We used the $\mathrm{mK}$ facilities of the Kamerlingh Onnes Laboratory at the University of Leiden to perform the shown measurements.

All chips were mounted inside a single module made of Niobium. The measurements below $4.2 \mathrm{~K}$ were done in a dilution refrigerator.

For the readout we used commercial available direct coupled electronics. For the measurements on the flux noise, we always used the FLL mode of the electronics and took the values in the white noise frequency region. All following given values of the energy resolution were calculated using the SQUID inductance $L_{S Q}$ estimated during the design. The values for the input current resolution are based on the measured mutual inductance $M$.

\section{B. Properties of the dc-SQUID With Integrated Flux Transformer}

The measured flux-voltage characteristics of the SQUID at two different temperatures are shown in Fig. 3. Above the shown 
voltage range, at $60 \mu \mathrm{V}$, there is a bias point without modulation, corresponding reasonably good to the resonance frequency of the washer with the pickup coils. Using an approximation from [9], this frequency was guessed and corresponds to a voltage of $45 \mu \mathrm{V}$.

At $4.2 \mathrm{~K}$, we observed a maximum flux-voltage transfer of 2.5 times the value calculated with the theory for non hysteretic, uncoupled dc-SQUIDs [6], [7]: $110 \mu \mathrm{V} / \Phi_{0}$. This transfer even increased at lower temperatures, 7.6 times higher transfer at $0.3 \mathrm{~K}$.

The mutual inductance of the signal coil system was $8.7 \mathrm{nH}$ instead of the design value of $10.9 \mathrm{nH}$.

A minimum flux noise of $1.4 \mu \Phi_{0} / \sqrt{ } \mathrm{Hz}$ was measured at $4.2 \mathrm{~K}$ in a two-stage setup with a DROS as second stage. Because of the large transfer function at low temperatures we did not achieve to get a two-stage setup working. On the other hand, at lower temperatures we were able to measure the noise in a single stage current biased configuration. A constant minimum flux noise of $2.8 \mu \Phi_{0} / \sqrt{ } \mathrm{Hz}$ was achieved at temperatures below $1 \mathrm{~K}$ and because of the steeper characteristics this value was not dominated by the input noise of the room temperature electronics, although there is still some contribution. Our rough guess of the real flux noise below $1 \mathrm{~K}$ is $1.5-2 \mu \Phi_{0} / \sqrt{ } \mathrm{Hz}$, subtracting the flux noise PSD related to the room temperature amplifier from the measured value. The contribution of the room temperature amplifier was estimated using the fluxvoltage transfer and the input voltage noise of the electronics. This would be higher compared to the noise at $4.2 \mathrm{~K}$. The reason for this behavior is most probably the non dc-SQUID-like behavior of temperature dependent characteristics.

\section{Properties of the dc-SQUID With a Parallel Washer Configuration}

The characteristics of the dc-SQUID with a parallel washer configuration at two different temperatures are shown in Fig. 4. The resonance of the washer of the signal coil is again in good agreement to a point of no modulation at $50 \mu \mathrm{V}$. From the design we guessed that this resonance occurs at $52 \mu \mathrm{V}$ [9]. Below this point there are basically two fields of operation. At voltages smaller than $20 \mu \mathrm{V}$, the characteristics are again very steep and getting steeper with lower temperatures. At for example $0.6 \mathrm{~K}$, this operation range was unusable in the two-stage setup.

In the operation range above about $20 \mu \mathrm{V}$, the transfer is also increasing with decreasing temperature, but with reasonable values. At $0.6 \mathrm{~K}$ the flux-voltage transfer is in the same range as the one calculated from theory [6], [7]: $70 \mu \mathrm{V} / \Phi_{0}$.

Because of its high transfer, especially at lower temperatures, we used the dc-SQUID with flux transformer as the second stage to characterize the SQUID with the parallel washer configuration. The first stage was voltage biased with a resistor of $0.5 \Omega$. To reduce negative effects of wideband noise in the second stage, we used standard ferrite-based inductors of in total $60 \mu \mathrm{H}$ as filter between the first and the second stage. In FLL, we reached typical bandwidths of above $50 \mathrm{kHz}$. In future configurations, the high inductance can of course still be reduced to gain more bandwidth and slew rate.

At $4.2 \mathrm{~K}$, the minimum measured noise flux noise was $2.1 \mu \Phi_{0} / \sqrt{ } \mathrm{Hz}$. The working point was in the steep part of the flux-voltage characteristics as shown in Fig. 4 at a bias current
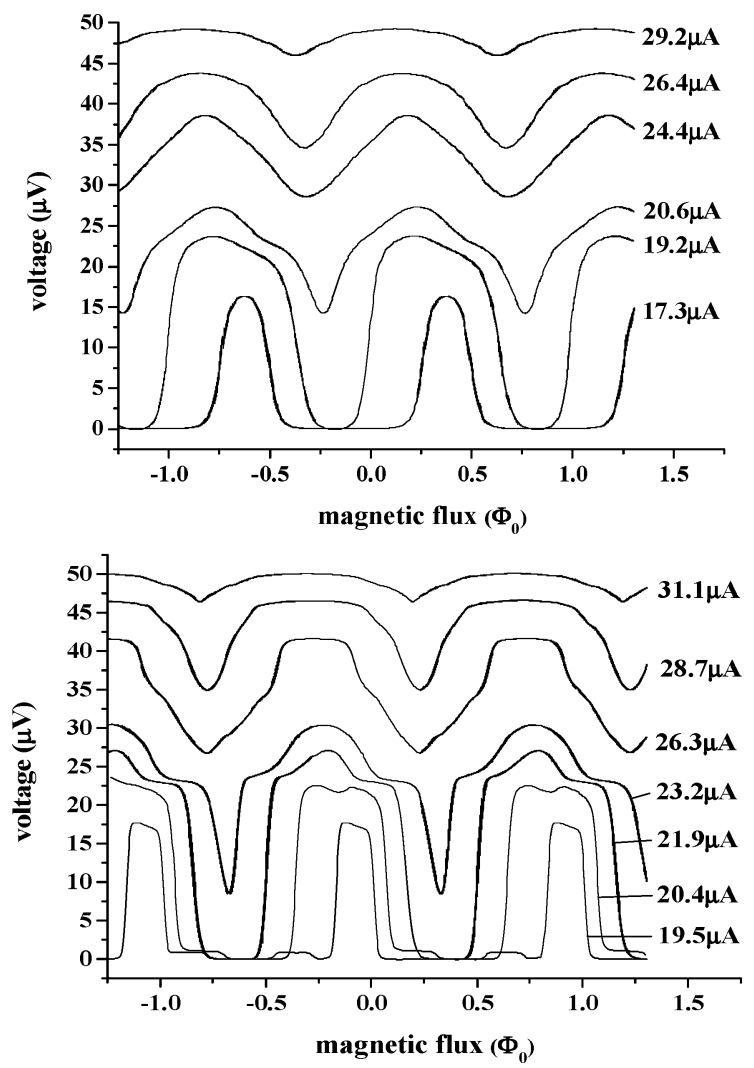

Fig. 4. Characteristics measured with sweeping magnetic flux measured in single stage with current bias at $4.2 \mathrm{~K}$ (upper) and at $0.6 \mathrm{~K}$ (lower). The applied bias currents are indicated on the right side of the graphs. A constant flux bias was applied. The signal coil was shunted with a resistor of $50 \Omega$ and a capacitor of $1 \mathrm{nF}$ in series. For the lower working range, at $4.2 \mathrm{~K}$ the maximum flux-to-voltage transfer was estimated to be $220 \mu \mathrm{V} / \Phi_{0}$ (at $19.2 \mu \mathrm{A}$ ) and at 0.6 $\mathrm{K} 440 \mu \mathrm{V} / \Phi_{0}($ at $20.4 \mu \mathrm{A})$. For the higher working range we estimated at 4.2 $\mathrm{K}$ a transfer of $38 \mu \mathrm{V} / \Phi_{0}$ (at $26.3 \mu \mathrm{A}$ ) and at $0.6 \mathrm{~K}$ of $75 \mu \mathrm{V} / \Phi_{0}$.

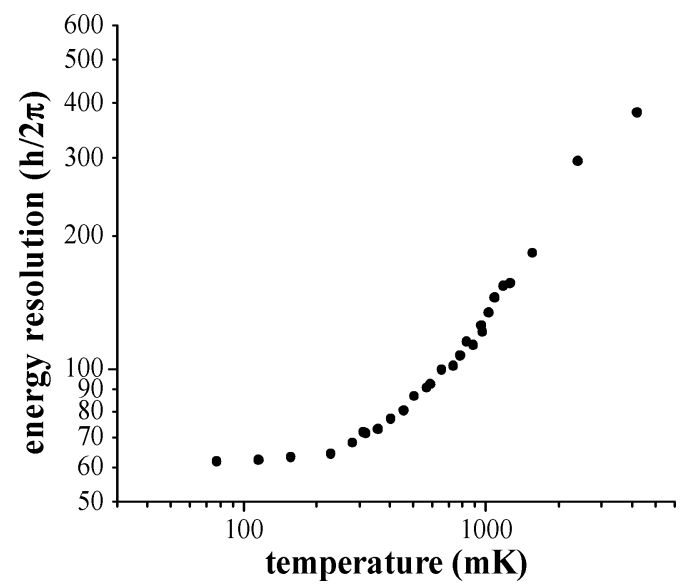

Fig. 5. The measured minimum sensitivity of the dc-SQUID with a parallel washer configuration measured at different temperatures. The energy resolution was calculated from the measured flux-noise using the SQUID inductance estimated during the design process $(233 \mathrm{pH})$.

of $26.4 \mu \mathrm{A}$. The energy resolution is estimated to be $380 \hbar$, which is much higher than the value calculated during design for a standard SQUID of $89 \hbar$.

The measured sensitivity at this working point also for lower temperatures is shown in Fig. 5. Below $200 \mathrm{mK}$ we reached a minimum energy resolution of nominally $62 \hbar$, which was 
calculated from the measured value of $0.84 \mu \Phi_{0} / \sqrt{ } \mathrm{Hz}$. With the measured mutual inductance between the signal coil system and the SQUID inductance $M$ of $11.2 \mathrm{nH}$, the equivalent noise on the signal current line is $155 \mathrm{fA} / \sqrt{ } \mathrm{Hz}$.

During the measurement, we observed a temperature gradient between the used thermometer resistance and the actual temperature within our SQUID system. The temperature shown in Fig. 5 was calculated using the flux noise in the second stage, while the first stage was switched off. This noise originates from the Johnson noise of the bias resistor and its PSD is thus proportional to the temperature.

\section{INTERPRETATION OF THE RESULTS}

First we observed steeper flux-voltage characteristics than predicted by the theory for standard dc-SQUIDs. Further the characteristics are getting less steep and smoother at higher temperature. This can be explained by wideband flux noise of thermal origin, the Johnson noise in the shunt- and damping-resistors.

The origin of this deviating behavior could most probably be found in parasitic capacitances introduced by the integrated coils and the influence of the washer resistance, analysed for example in [10]. We have a few indications that the washer resistance causes this unexpected behavior.

First there are no clear resonance effects in the operation range. The coil resonances are located below, which follows from the big length of the coil, and the washer resonances above the operation range, seen experimentally. The transition-voltage range in the characteristics of the dc-SQUID with a parallel washer configuration around $20 \mu \mathrm{V}$ still shows modulation, so at least no strong resonance point.

Second, there is no clear double-loop structure separating the SQUID inductance into two parts, separated by the washer resistance and the parasitic capacitance introduced by the integrated coil. The washer resistor in both designs is directly connected to the junctions and its presence can change the characteristics [10]. The loop formed by the junctions and the washer resistance is especially in the case of the dc-SQUID with flux transformer very small. It is, except for the normal slit in the washer, completely on top of a Niobium layer.

In case of the dc-SQUID with the parallel washer configuration, the expected inductance connected to the washer resistance should be much higher. Because of the connection of four washers and the series connection of the feedback and signal coils, the loop containing the washer resistance and the Josephson Junctions is crossing six slits in the niobium layer below. An inductance in series with the washer resistance could be an explanation for the changes in behavior at higher voltages, so higher oscillation frequencies. Here, the damping impedance should be less effective. An inductance of $100 \mathrm{pH}$ in series with the damping resistance of $5.6 \Omega$ would become predominant imaginary above Josephson frequencies corresponding to $20 \mu \mathrm{V}$, the transition voltage to the low-noise operation range. Numerical inductance calculations using Fasthenry [11] on a simplified geometry suggested an inductance of $180 \mathrm{pH}$ in the loop of the damping resistance and the Josephson junctions.

We are working on numerical simulations including parasitic elements to find an explanation for the measured characteristics.

\section{CONCLUSION}

We have designed two sensor SQUIDs for the gravitational wave antenna MiniGRAIL. The fabricated samples fit the design criteria, but during characterization we found big differences to the expected behavior. Both designs showed steeper characteristics and a degraded sensitivity, which can not be explained by the standard dc-SQUID theory. In a special region of operation, the dc-SQUID with a parallel washer configuration shows a reasonable sensitivity. The noise corresponds to a white current noise of $155 \mathrm{fA} / \sqrt{ } \mathrm{Hz}$ through the input coil.

To explain the behavior of the designed SQUIDs, we now focus on numerical simulations also including parasitic elements introduced by the input coils.

\section{REFERENCES}

[1] A. De Waard, L. Gottardi, J. Van Houwelingen, A. Shumack, and G. Frossati, "MiniGRAIL, the first spherical detector," Class. Quantum Grav., vol. 20, pp. S143-S151, Apr. 2003.

[2] F. C. Wellstood, C. Urbina, and J. Clarke, "Hot-electron limitation to the sensitivity of the dc superconducting quantum interference device," Appl. Phys. Lett., vol. 54, pp. 2599-2601, June 1989.

[3] Vinante, M. Bonaldi, P. Falferi, M. Cerdonio, R. Mezzena, G. A. Prodi, and S. Vitale, "Stabilization and optimization of a two-stage dc SQUID coupled to a high Q resonator," Physica C, vol. 368, pp. 176-180, Mar. 2002.

[4] IPHT Jena e. V. Department of Quantum Electronics, Winzerlaer Str. 10, 07745 Jena, Germany, Homepage http://www.ipht-jena.de.

[5] M. Podt, L. Gottardi, A. De Waard, G. Frossati, and J. Flokstra, "Development of a SQUID Readout System for the MiniGRAIL," IEEE Trans. Appl. Supercond., vol. 15, pp. 785-788, June 2005.

[6] C. D. Tesche and J. Clarke, "DC SQUID: Noise and optimization," J. Low Temp. Phys., vol. 29, pp. 301-331, Nov. 1977.

[7] J. J. P. Bruines, V. J. de Waal, and J. E. Mooij, "Comment on "DC SQUID: Noise and optimization" by Tesche and Clarke," J. Low Temp. Phys., vol. 46, pp. 383-386, Feb. 1982.

[8] M. B. Simmonds, "High Symmetric dc SQUID System," US Patent No. 5053834, 1991.

[9] K. Enpuku, R. Cantor, and H. Koch, "Modeling the dc superconducting quantum interference device coupled to the multiturn input coil. III," $J$. Appl. Phys., vol. 72, pp. 1000-1006, Aug. 1992.

[10] T. Ryhänen and H. Seppä, "Effect of parasitic capacitance and inductance on the dynamics and noise of dc superconducting quantum interference devices," J. Appl. Phys., vol. 71, pp. 6150-6166, Jun. 1992.

[11] Inductance Extraction Software: FastHenry 3.0wr. Whiteley Research Inc., 456 Flora Vista Avenue, Sunnyvale, CA 94086, U.S.A., http:// www.srware.com. 